\title{
Validation through comparison: Measurement and calculation of the bistatic radar cross section of a stealth target
}

\author{
L. Gürel and H. Bağc1 \\ Department of Electrical and Electronics Engineering, Bilkent University, Ankara, Turkey
}

\author{
J. C. Castelli, A. Cheraly, and F. Tardivel
}

Electromagnetism and Radar Department, Office National d'Etudes et de Recherche Aérospatiale, Châtillon Cedex, France

Received 30 November 2001; revised 2 December 2002; accepted 20 February 2003; published 3 June 2003.

[1] Bistatic radar cross section (BRCS) values of a stealth airborne target are predicted by performing both scaled-model measurements and numerical simulations. In order to achieve the solution of large-scale electromagnetic problems in the numerical simulation environment, the fast multipole method (FMM) is implemented and used. The FMM has produced remarkably accurate results, in addition to its efficiency. The efficiency of the FMM is due to its reduced computational complexity and memory requirement, which are both $O\left(N^{1.5}\right)$. Comparison of the measured and computed BRCS values has resulted in a striking agreement, which serves to validate both of the prediction techniques. INDEX TERMS: 0634 Electromagnetics: Measurement and standards; 0619 Electromagnetics: Electromagnetic theory; 0644 Electromagnetics: Numerical methods; 0669 Electromagnetics: Scattering and diffraction; KEYWORDS: electromagnetic scattering, radar cross section, fast multipole method, stealth, RCS computations, RCS measurements

Citation: Gürel, L., H. Bağcı, J. C. Castelli, A. Cheraly, and F. Tardivel, Validation through comparison: Measurement and calculation of the bistatic radar cross section of a stealth target, Radio Sci., 38(3), 1046, doi:10.1029/2001RS002583, 2003.

\section{Introduction}

[2] In many civilian and almost all military applications, an accurate knowledge of the radar cross section (RCS) [Stone, 1989; Knott, 1993; Bhattacharyya and Sengupta, 1991; Skolnik, 1991] of the involved targets is highly desirable. The RCS information is used for numerous purposes, ranging from the design of novel stealth vehicles with reduced radar signatures [Ufimtsev, 1996] to the decision of what kind of electronic counter measures $(\mathrm{ECM})$ to engage against a certain threat [Winchester, 1992].

[3] RCS is a function of various parameters, such as the frequency, directions of incidence and scattering, and polarization. When the directions of incidence and the scattering are the same, the resulting RCS values are called monostatic RCS. Bistatic RCS (BRCS) [Glasier, 1989; Blyakhman and Runova, 2001] is due to the more general case, namely, when the directions of incidence and scattering are not necessarily the same. This is the

Copyright 2003 by the American Geophysical Union. 0048-6604/03/2001RS002583 kind of RCS obtained by a bistatic radar that uses antennas at different locations for transmission and reception [Hanle, 1986]. The concept of bistatic radar is not new, since the earliest radars were of this type. However, recently there is renewed interest in bistatic radars due to their ability to detect stealth targets [Boyle and Wasylkiwskyj, 1994]. Furthermore, bistatic and passive radars employing transmitters of opportunity, such as television broadcasting transmitters [Howland, 1999; Poullin and Lesturgie, 1994], have recently emerged as exciting possibilities. A good understanding of the BRCS that is obtained by various types of bistatic radars for different targets is imperative for the development and optimization of such radars.

[4] Another area, where the accurate determination of the RCS of targets is important, is the design of stealth targets [Ufimtsev, 1996]. It is clear that reduction of RCS cannot be accomplished without a tool to determine the RCS by using actual measurements or prediction techniques.

[5] A direct measurement of the RCS may not always be possible either due to the economics of the measurement campaign or simply because the target may not exist yet. In that case, prediction of the RCS becomes the 


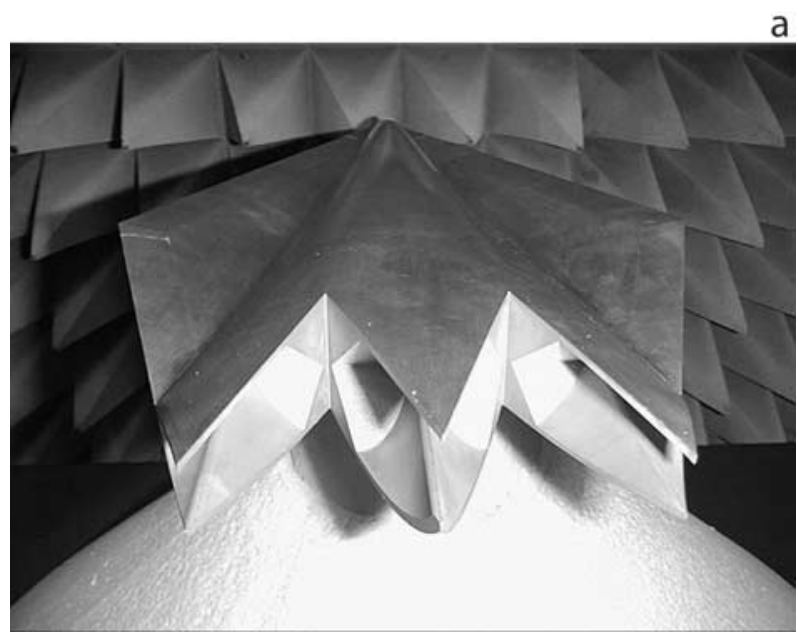

a
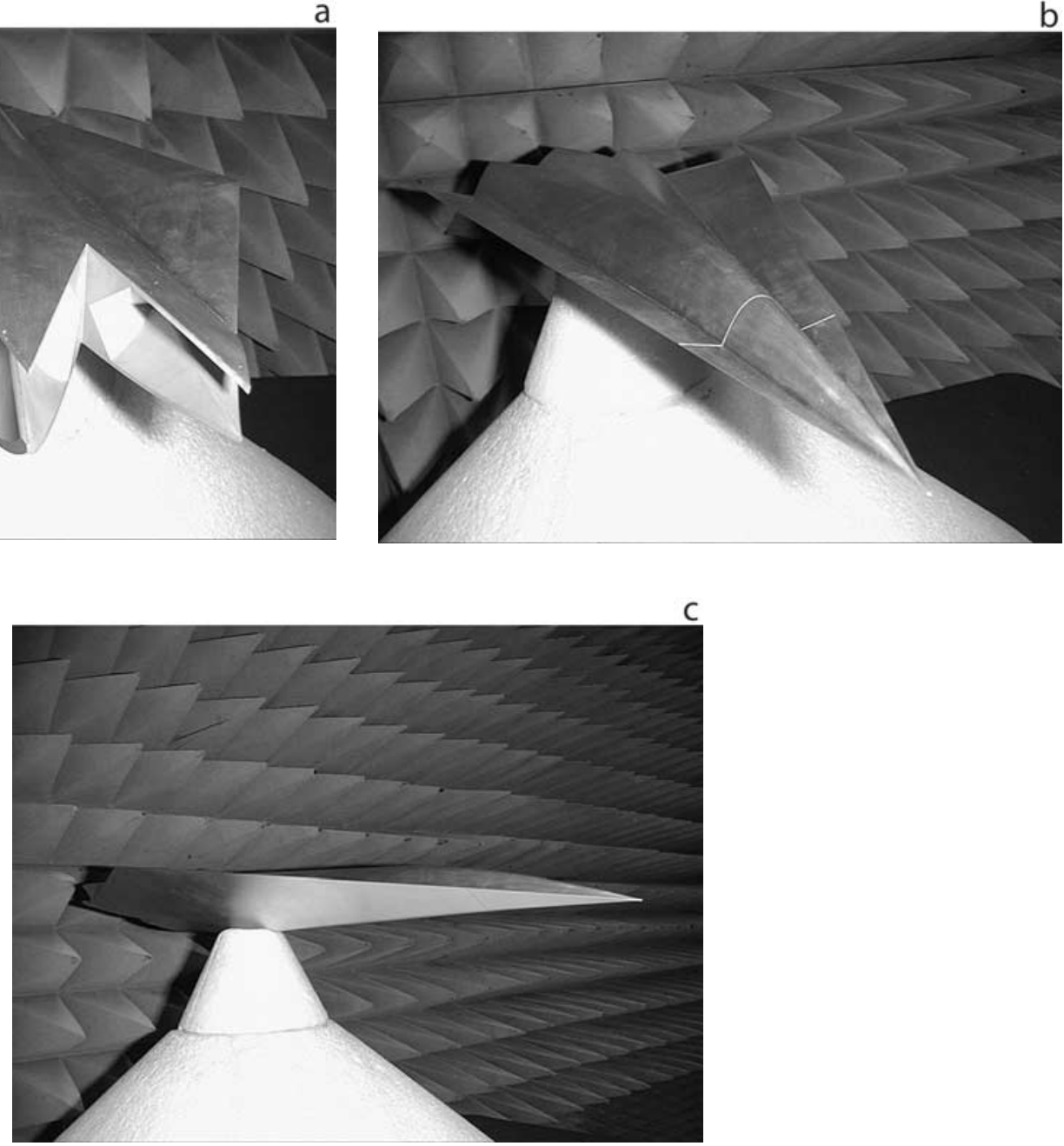

Figure 1. Photographs of the scaled model of the FLAMME stealth airborne vehicle used in bistatic radar cross section (BRCS) measurements and computations: (a) aft view, (b) front view, and (c) side view.

preferred means of obtaining this vital information. However, it is of utmost importance that the predicted values be accurate enough to successfully replace the actual RCS values.

[6] RCS prediction can be achieved through numerical calculations or scaled-model measurements. In this paper, the use of both of these techniques to predict the RCS of a stealth airborne target is reported. The scaled-model measurements are performed at the Office National d'Etudes et de Recherche Aérospatiales (ONERA), France. The numerical simulations are performed at Bilkent University, Turkey, where the fast multipole method (FMM) [Rokhlin, 1990; Coifman et al., 1993; Song et al., 1997; Sheng et al., 1998] is used as the numerical solver of choice in order to efficiently solve large computational- electromagnetics (CEM) problems without sacrificing the accuracy of the results. The two prediction campaigns are carried out independently, and the results are documented internally before they are exchanged with the other institution. The two sets of results, which are not calibrated to force them to match, will be compared to demonstrate the good agreement with each other. It will be argued that the good agreement of the two sets of results testifies to the accuracy of both the numerical computations and the scaled-model measurements.

\section{Stealth Target}

[7] In this work, our aim is to compare the results of BRCS measurements and computations performed for 


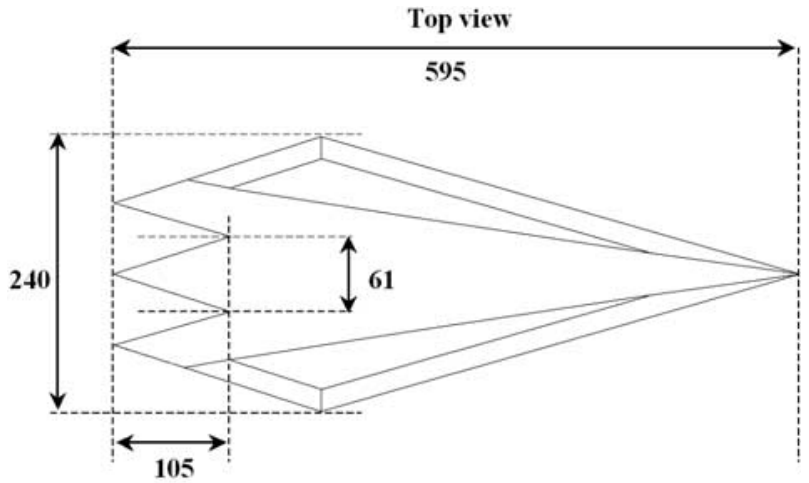

Side view

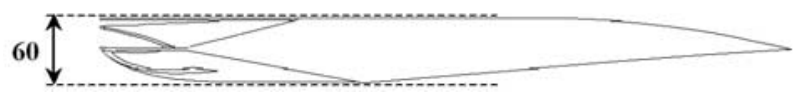

Figure 2. The dimensions of the FLAMME scaled model in millimeters.

the same target. For this purpose, a scaled model of stealth airborne target, called FLAMME, is selected. The FLAMME was designed and built by ONERA for the 1993 Paris Air Show exhibition as an unclassified demonstration of a possible future stealth cruise missile or drone. Photographs of a scaled model of this stealth airborne vehicle are shown in Figure 1. A sketch displaying the dimensions of this 1:10 model is illustrated in Figure 2. The FLAMME scaled model is made of solid conducting material and does not include any dielectric or radar absorbing materials. The shape of this stealth vehicle was designed in order to achieve a low monostatic RCS of the full-scale target at centimetric wavelengths. The $\mathrm{V}$ shape of the wings allows the incident waves to reflect in downward and bistatic directions and to hide the above part of the body, where various equipment, such as communication antennas, can be installed. The aft part of the body is jagged in order to cancel the scattering of surface waves in the monostatic front direction. The top view of the FLAMME scaled model (Figure 2) shows that the projections of all leading and trailing edges of the wings on a horizontal plane are oriented along only two directions. These two directions are called the "dustbin" directions. In the horizontal plane, the monostatic RCS of the scaled model is concentrated around the four directions that are perpendicular to the two dustbin directions. Therefore the monostatic RCS values of FLAMME are quite high with narrow beam widths around these four directions. Two of these four directions are perpendicular to the leading edges of the wings, which are about $45 \mathrm{~cm}$ long and constitute the dominant mechanisms of scattering from the FLAMME. The length of the trailing edges of the wings is much shorter. Therefore scattering from the trailing edges is secondary compared to the leading edges of the wings.

[8] ONERA performed the BRCS measurements in its anechoic facility "BABI" (Figure 3 ) on the scaled model of the FLAMME in the $2-4 \mathrm{GHz}$ frequency range. Details of the measurement campaign are given in section 3. The group in Bilkent University carried out the BRCS computations of the same stealth target at the same frequencies, as explained in section 4 .

\section{Measurements}

[9] The facility at ONERA that is used for the BRCS measurements of the scaled FLAMME model is called BABI (French acronym for bistatic facility) [Castelli, 1994]. This facility is capable of transmitting and receiving coherent $\mathrm{CW}$ signals in horizontal and vertical polarizations from $1 \mathrm{GHz}$ up to $18 \mathrm{GHz}$ by using an HP 8510 network analyzer system. The target-antenna distance is constant at $5.5 \mathrm{~m}$ during all the measurements. Since the largest scattering feature of the FLAMME scaled model (which is the leading edge of the wing) is $0.45 \mathrm{~m}$ long, this target-antenna distance comfortably allows a farfield configuration up to $4 \mathrm{GHz}$.

[10] The measurements of FLAMME scaled model are realized with two bipolarized wideband horn antennas that are used as transmitting and receiving antennas. Using these bipolarized antennas, the copolar and cross-polar components of the complex scattering matrix

$$
\overline{\mathbf{Z}}=\left[\begin{array}{ll}
Z_{\mathrm{HH}} & Z_{\mathrm{HV}} \\
Z_{\mathrm{VH}} & Z_{\mathrm{VV}}
\end{array}\right]
$$

of the FLAMME can be measured by employing background subtraction. However, it should be noted

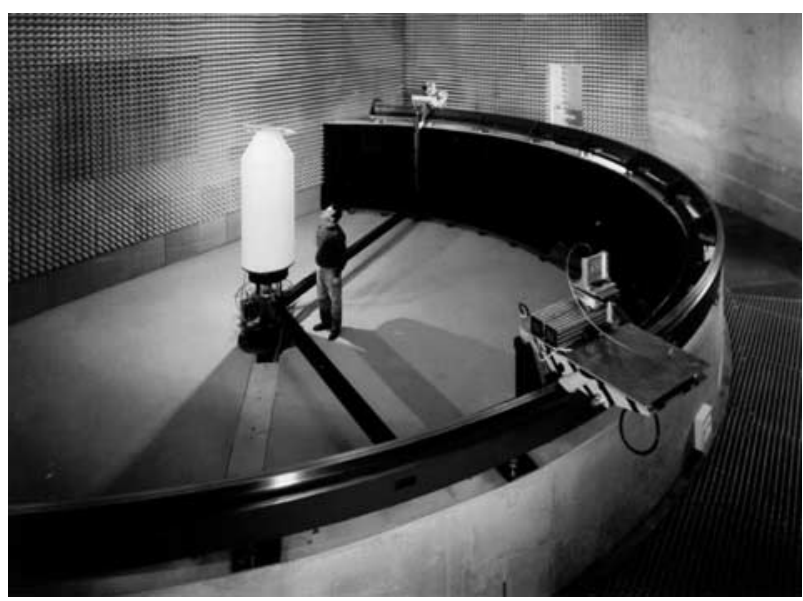

Figure 3. ONERA bistatic anechoic measurement facility "BABI." 
that the measured scattering matrix $\overline{\mathbf{Z}}$ is not the same as the desired positive real BRCS matrix

$$
\overline{\boldsymbol{\sigma}}=\left[\begin{array}{ll}
\sigma_{\mathrm{HH}} & \sigma_{\mathrm{HV}} \\
\sigma_{\mathrm{VH}} & \sigma_{\mathrm{VV}}
\end{array}\right]
$$

of the FLAMME, which is equivalent to its theoretical counterpart formulated in section 4 . We first have to perform a polarimetric calibration of the scattering matrix $\overline{\mathbf{Z}}$ to obtain the calibrated scattering matrix

$$
\overline{\mathbf{S}}=\left[\begin{array}{ll}
\mathrm{S}_{\mathrm{HH}} & \mathrm{S}_{\mathrm{HV}} \\
\mathrm{S}_{\mathrm{VH}} & \mathrm{S}_{\mathrm{VV}}
\end{array}\right]
$$

and consequently the theoretical RCS matrix $\overline{\mathbf{s}}$. This is achieved by using two calibration targets, namely, a sphere and a dihedral corner. Let $Z_{\mathrm{HH}}^{\mathrm{SPH}}$ and $\mathrm{Z}_{\mathrm{VV}}^{\mathrm{SPH}}$ denote the complex measured scattering data of the sphere, $Z_{\mathrm{HV}}^{\mathrm{DC}}$ and $\mathrm{Z}_{\mathrm{VH}}^{\mathrm{DC}}$ denote the complex measured scattering data of the dihedral corner, and $\mathrm{S}^{\mathrm{SPH}}$ be the theoretical value of the complex backscattering coefficient of the sphere, which is calculated by the Mie-series solution [Mie, 1908] and related to the monostatic RCS of the sphere through $\sigma^{\mathrm{SPH}}=\left|\mathrm{S}^{\mathrm{SPH}}\right|^{2}$. Then, the calibrated scattering coefficients of the FLAMME are given by [Sarabandi et al., 1990]

$$
\begin{gathered}
\mathrm{S}_{\mathrm{HH}}=\frac{\mathrm{S}^{\mathrm{SPH}}}{\mathrm{Z}_{\mathrm{HH}}^{\mathrm{SPH}}} \mathrm{Z}_{\mathrm{HH}}, \\
\mathrm{S}_{\mathrm{HV}}=\frac{\mathrm{S}^{\mathrm{SPH}} \mathrm{Z}_{\mathrm{HV}}}{\sqrt{\mathrm{Z}_{\mathrm{HH}}^{\mathrm{SPH}} \mathrm{Z}_{\mathrm{VV}}^{\mathrm{SPH}}}} \sqrt{\frac{Z_{\mathrm{VH}}^{\mathrm{DC}}}{Z_{\mathrm{HV}}^{\mathrm{DC}}}} \\
\mathrm{S}_{\mathrm{VH}}=\frac{\mathrm{S}^{\mathrm{SPH}} \mathrm{Z}_{\mathrm{VH}}}{\sqrt{\mathrm{Z}_{\mathrm{HH}}^{\mathrm{SPH}} \mathrm{Z}_{\mathrm{VV}}^{\mathrm{SPH}}}} \sqrt{\frac{\mathrm{Z}_{\mathrm{HV}}^{\mathrm{DC}}}{\mathrm{Z}_{\mathrm{VH}}^{\mathrm{DC}}}}, \\
\mathrm{S}_{\mathrm{VV}}=\frac{\mathrm{S}^{\mathrm{SPH}}}{\mathrm{Z}_{\mathrm{VV}}^{\mathrm{SPH}}} \mathrm{Z}_{\mathrm{VV}} .
\end{gathered}
$$

Finally, the desired BRCS matrix is computed as

$$
\mathbf{s}=\left[\begin{array}{ll}
\left|\mathrm{S}_{\mathrm{HH}}\right|^{2} & \left|\mathrm{~S}_{\mathrm{HV}}\right|^{2} \\
\left|\mathrm{~S}_{\mathrm{VH}}\right|^{2} & \left|\mathrm{~S}_{\mathrm{VV}}\right|^{2}
\end{array}\right]
$$

With the help of the software that controls the measurements and analyzes the measured data according to the isolated-antenna calibration technique [Sarabandi et al., 1990; Titin-Schnaider et al., 1994] outlined in equations (1)-(8), it is also possible to obtain the full coherent polarization matrix of the BRCS values in the $\mathrm{HH}, \mathrm{VV}, \mathrm{HV}$, and $\mathrm{VH}$ configurations.

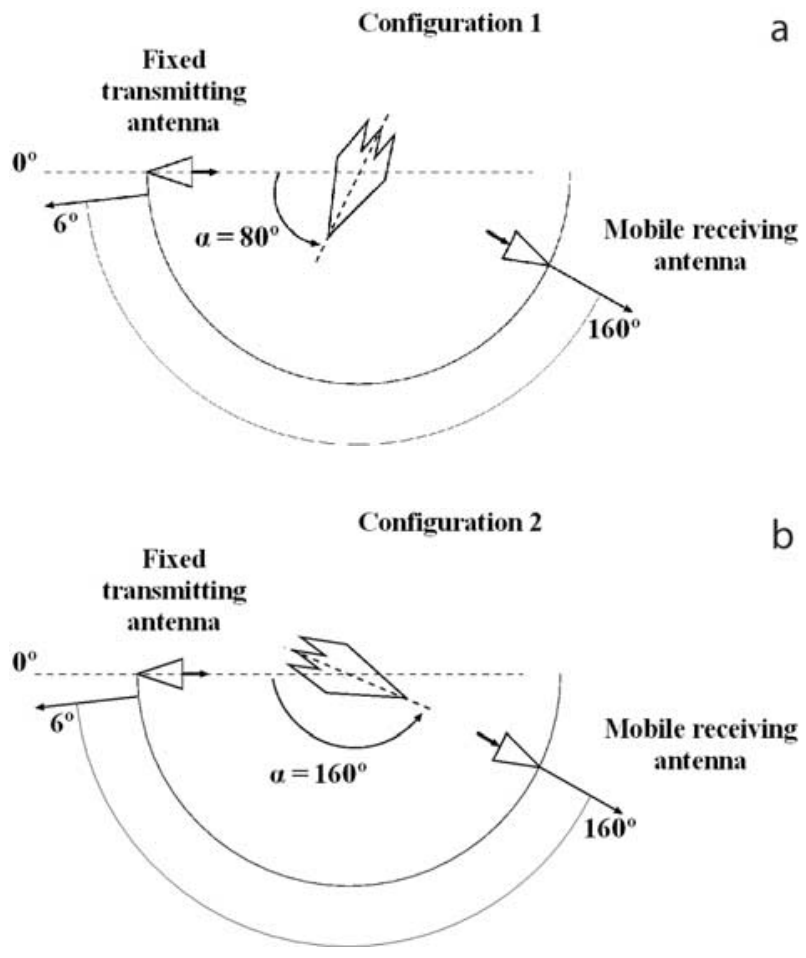

Figure 4. Measurement configurations.

[11] The BRCS measurements are performed for two different values of the angle between the axis of the model and the fixed transmitting antenna. This angle is represented by $\alpha$ in Figure 4, where it is set to $80^{\circ}$ and $160^{\circ}$, respectively, for the two measurement configurations. Another parameter for the measurement configurations is the angle between the fixed transmitting antenna and the mobile receiving antenna. This bistatic angle, which is denoted with $\beta$, changes from $6^{\circ}$ and $160^{\circ}$ by discrete steps of $0.5^{\circ}$ in both of the configurations as shown in Figure 4. In both of the configurations, the pitch and the roll of the scaled model are $0^{\circ}$ so that the planar portion of the top surface of the model becomes parallel to the antenna plane, as illustrated in Figure 5. The measurements are carried out from 2 to $4 \mathrm{GHz}$ with a frequency step of $25 \mathrm{MHz}$. For each illumination at a fixed $\alpha$, the frequency and $\beta$ sweeps are repeated twice to obtain first the $\mathrm{HH}$ and $\mathrm{HV}$, and then the VH and VV coherent BRCS values. Measured RCS values of the FLAMME will be presented in section 5 in comparison to the computed results.

\section{Computations}

[12] Employing numerical simulations for RCS computations is a cost-effective and attractive alternative to 


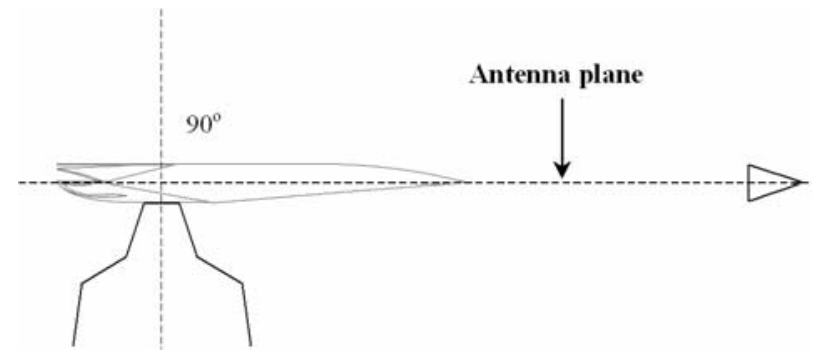

Figure 5. Relative position of the scaled model with respect to the antenna plane.

performing scaled-model measurements. However, difficulties arise as the target size becomes large compared to the wavelength since the electromagnetic problem translates into a large-scale computing problem. Solution of large-scale problems with traditional algorithms tends to consume the computational resources fast enough to prohibit accurate and meaningful solutions. Therefore fast and efficient algorithms that require reduced computational resources are needed to solve these challenging computational electromagnetics problems. For this purpose, the FMM is employed in the electromagnetics simulation environment developed in Bilkent University and used for the BRCS computations of the FLAMME stealth target described in section 2 .

[13] The FLAMME stealth airborne target, whose computer model is shown in Figure 6a, is electromagnetically modeled as a closed surface with a complicated geometry and made out of perfect electric conductor (PEC). When illuminated by an incident time-harmonic electromagnetic wave, a current density, denoted by $\mathbf{J}_{s}\left(\mathbf{r}^{\prime}\right)$, is induced on the surface of the PEC target. The scattered field is given by

$$
\mathbf{E}^{S}(\mathbf{r})=\int_{S} d S^{\prime} \overline{\mathbf{G}}\left(\mathbf{r}, \mathbf{r}^{\prime}\right) \cdot \mathbf{J}_{S}\left(\mathbf{r}^{\prime}\right),
$$

where $\overline{\mathbf{G}}\left(\mathbf{r}, \mathbf{r}^{\prime}\right)$ is the dyadic Green's function and $S$ denotes the surface of the target. The unknown surface current density $\mathbf{J}_{s}\left(\mathbf{r}^{\prime}\right)$ can be expanded using $N$ basis functions $\mathbf{b}_{j}\left(\mathbf{r}^{\prime}\right)$ and $N$ unknown coefficients $a_{j}$ as

$$
\mathbf{J}_{S}\left(\mathbf{r}^{\prime}\right)=\sum_{j=1}^{N} \mathbf{b}_{j}\left(\mathbf{r}^{\prime}\right) a_{j} .
$$

The basis functions are defined as Rao-Wilton-Glisson (RWG) basis functions [Rao et al., 1982; Gürel et al., 1999], which are piecewise linear functions defined on triangular domains. Figure $6 \mathrm{~b}$ shows the meshed model of the target surface, which provides the triangulated surface required for the use of the RWG basis functions. In obtaining this triangulation, the model surface is "discretized" by planar triangular facets with edges not longer than one tenth of the wavelength. Since the tangential component of the total electric field must vanish everywhere on the PEC surface, we obtain the electric-field integral equation (EFIE)

$$
\begin{aligned}
& \int_{S_{i}} d S \mathbf{t}_{i}(\mathbf{r}) \cdot \mathbf{E}^{S}(\mathbf{r})=-\int_{S_{i}} d S \mathbf{t}_{i}(\mathbf{r}) \cdot \mathbf{E}^{i}(\mathbf{r}) \\
& \quad \text { for } i=1, \ldots, N .
\end{aligned}
$$

where $\mathbf{E}^{i}(\mathbf{r})$ is the incident electric field and $\mathbf{t}_{i}(\mathbf{r})$ is a testing function with vector components tangential to the surface. By defining $N$ such testing functions, equation (11) can be transformed into a matrix equation

$$
\overline{\mathbf{Z}}_{N \times N} \cdot \mathbf{a}_{N \times 1}=\mathbf{v}_{N \times 1}
$$
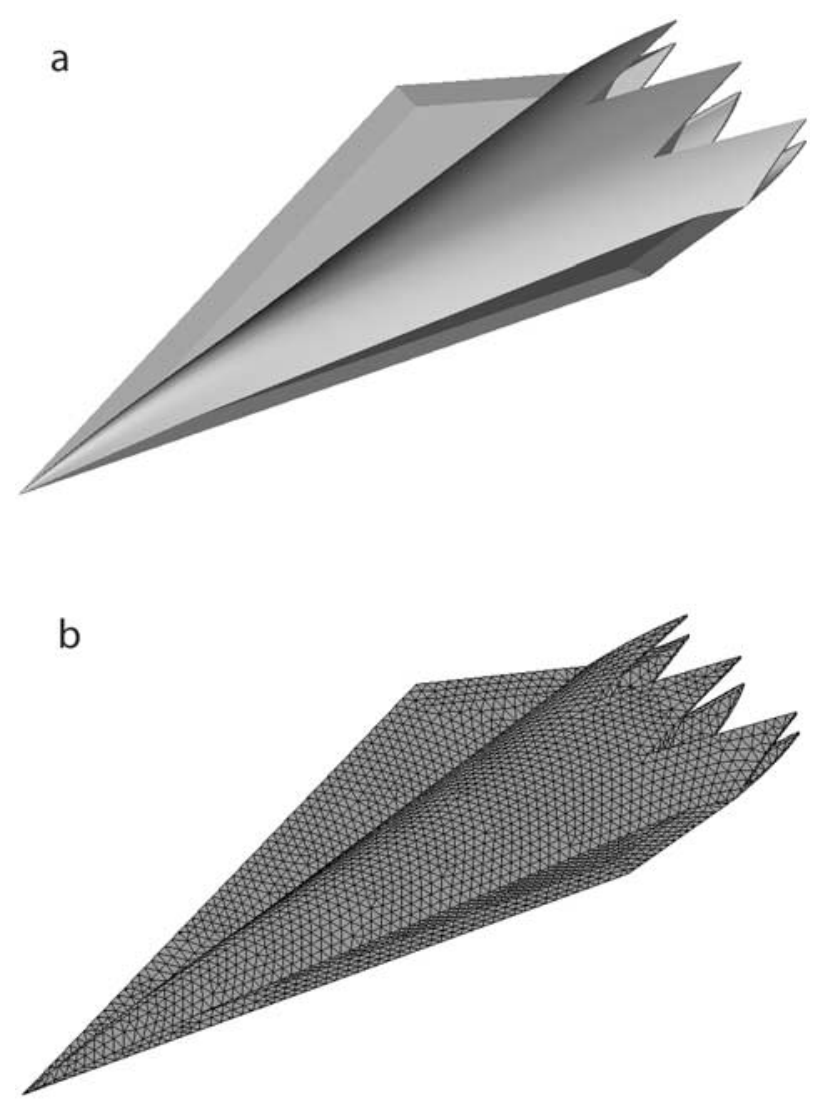

Figure 6. (a) Computer model of the stealth airborne target. (b) Meshed model of the target surface. 
where the elements of the "impedance" matrix and the right-hand-side vector are given as

$$
\begin{gathered}
Z_{i j}=\int_{S_{i}} d S \mathbf{t}_{i}(\mathbf{r}) \cdot \int_{S_{j}} d S^{\prime} \overline{\mathbf{G}}\left(\mathbf{r}, \mathbf{r}^{\prime}\right) \cdot \mathbf{b}_{j}\left(\mathbf{r}^{\prime}\right) \\
v_{i}=-\int_{S_{j}} d S \mathbf{t}_{i}(\mathbf{r}) \cdot \mathbf{E}^{S}(\mathbf{r}) .
\end{gathered}
$$

For the choice of the testing functions, the Galerkin method is used, where $\mathbf{t}_{i}(\mathbf{r})=\mathbf{b}_{i}(\mathbf{r})$ for all $i=1, \ldots, N$.

[14] A direct solution of the $N \times N$ linear system of equations in equation (12) requires $O\left(N^{3}\right)$ operations. In this work, equation (12) is solved by using an iterative solution method that is based on the minimization of a residual error vector,

$$
\mathbf{r}^{(k)}=\mathbf{v}-\overline{\mathbf{Z}} \cdot \mathbf{a}^{(k)},
$$

where $\mathbf{a}^{(k)}$ represents the $k$ th guess to the solution. The direct matrix-vector multiplication in equation (15) requires $O\left(N^{2}\right)$ operations per iteration. This multiplication is replaced by the FMM, which has $O\left(N^{1.5}\right)$ complexity. FMM achieves this performance by grouping all the unknowns into clusters formed on the basis of the physical proximity and by manipulating the fields of the clusters instead of the fields of the individual unknowns. A reduction from $O\left(N^{2}\right)$ to $O\left(N^{1.5}\right)$ in memory requirement is also achieved with the use of the FMM. This is the result of the fact that the whole impedance matrix is not stored during the matrix-vector multiplication. The reduced computational complexity and memory requirement of the FMM enable the simulation and the solution of large-scale problems by using smaller computational resources. For the sake of brevity, details of the formulation and the implementation of the FMM are not given here, but can be found in the literature [Rokhlin, 1990; Coifman et al., 1993; Song et al., 1997; Sheng et al., 1998; Gürel and Băgcl, 2001].

[15] Once the unknown coefficients $\boldsymbol{a}_{j}$ are solved using the FMM, the induced surface electric current is determined as in equation (10), from which the scattered electric field $\mathbf{E}^{S}(\mathbf{r})$ can be computed as in equation (9). Then, the copolar and cross-polar components of the RCS are computed as

$$
\left[\begin{array}{cc}
\sigma_{H H} & \sigma_{H V} \\
\sigma_{V H} & \sigma_{V V}
\end{array}\right]=\lim _{r \rightarrow \infty} 4 \pi r^{2}\left[\begin{array}{l}
\left|\frac{\mathrm{E}_{H}^{s}(\mathbf{r})}{\mathrm{E}_{H}^{i}(\mathbf{r})}\right|^{2}\left|\frac{\mathrm{E}_{H}^{s}(\mathbf{r})}{\mathrm{E}_{V}^{i}(\mathbf{r})}\right|^{2} \\
\left|\frac{\mathrm{E}_{V}^{s}(\mathbf{r})}{\mathrm{E}_{H}^{i}(\mathbf{r})}\right|^{2}\left|\frac{\mathrm{E}_{V}^{s}(\mathbf{r})}{\mathrm{E}_{V}^{i}(\mathbf{r})}\right|^{2}
\end{array}\right],
$$

where the incident field $\mathbf{E}^{i}(\mathbf{r})$ is implemented as a plane wave and the scattered field $\mathbf{E}^{S}(\mathbf{r})$ is computed in the far zone of the target.
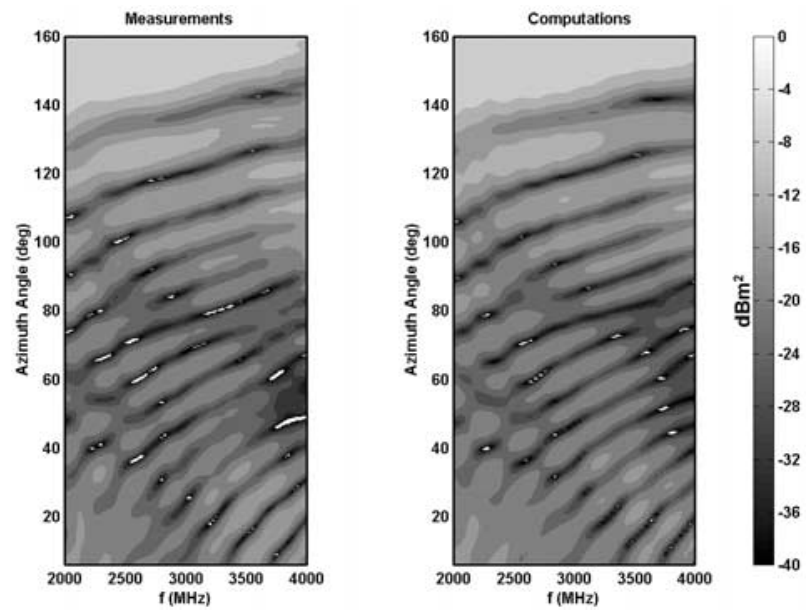

Figure 7. The HH-polarized measured and computed BRCS results for $\alpha=60^{\circ}$ incidence. See color version of this figure at back of this issue.

[16] The computed RCS results, which will be presented in section 5 in comparison to the measured results, are obtained for the two configurations described in Figure 4. The simulations are performed from 2 to $4 \mathrm{GHz}$ at every $25 \mathrm{MHz}$. Although the scattered fields can be computed at any arbitrary direction, the computed $\mathrm{RCS}$ data is presented for the bistatic angles of $6^{\circ}-160^{\circ}$ at every $0.5^{\circ}$ in order to match the range of the measured results. Computations at different frequencies are carried out using the same triangulation of the model as shown in Figure 6. This mesh is obtained by using a maximum subsection size of $0.75 \mathrm{~cm}$, which corresponds to one tenth of the wavelength at $4 \mathrm{GHz}$. This triangulation resulted in more than 12,000 unknowns for this threedimensional electromagnetic problem.

\section{Comparison of Results}

[17] In this section, the measured and computed BRCS results are presented and compared. It should be stressed that the measurements and the computations are performed completely independently by the ONERA group in France and the Bilkent University group in Turkey. Once the results are obtained, no artificial scaling or calibration is performed to force the results to match.

[18] Figures 7-14 present the measured and computed BRCS results for both of the incidence configurations described in Figure 4. Both copolarized ( $\mathrm{HH}$ and VV) and cross-polarized ( $\mathrm{HV}$ and $\mathrm{VH}$ ) $\mathrm{RCS}$ values are presented. Horizontal axes of all RCS plots denote the change of the frequency from 2 to $4 \mathrm{GHz}$, and the vertical axes correspond to the bistatic azimuth angle from $\beta=6^{\circ}$ to $\beta=160^{\circ}$, which is the angle between the transmitting and receiving antennas. Both measured and 

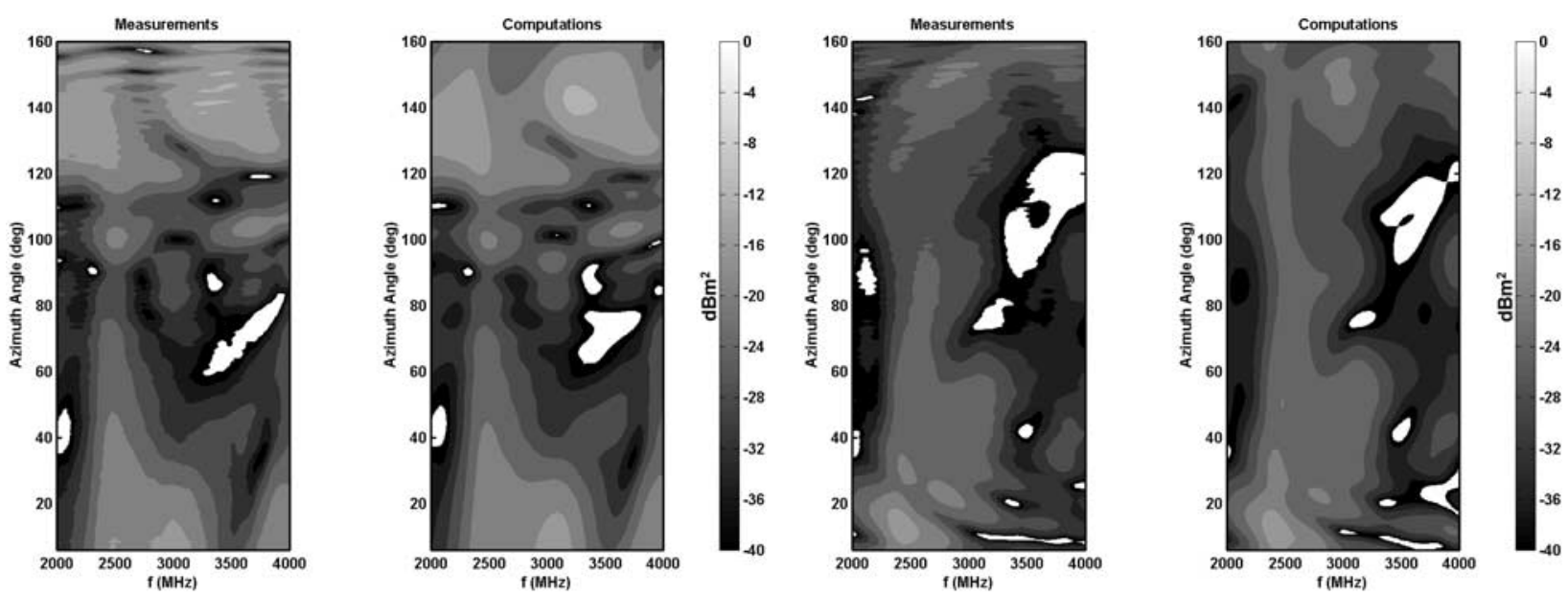

Figure 8. The VV-polarized measured and computed BRCS results for $\alpha=160^{\circ}$ incidence. See color version of this figure at back of this issue.

computed RCS values are presented in a logarithmic scale defined as

$$
\sigma\left[\mathrm{dBm}^{2}\right]=10 \log _{10} \sigma\left[\mathrm{m}^{2}\right] .
$$

Consequently, large and small values of RCS can be simultaneously examined and compared. In all plots, the BRCS values between 0 and $-40 \mathrm{dBm}^{2}$ are explicitly presented. The white areas in the contour plots correspond to the values of RCS less than $-40 \mathrm{dBm}^{2}$, which are not further detailed.

[19] An overall comparison of the measured and computed BRCS plots in Figures 7-14 indicates a remarkable agreement of the two sets of data. This observation
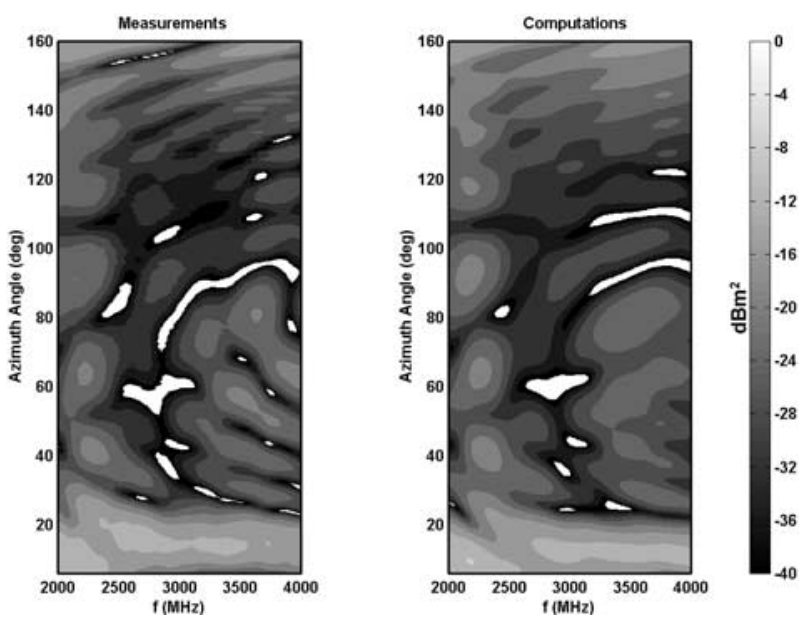

Figure 9. The HH-polarized measured and computed BRCS results for $\alpha=80^{\circ}$ incidence. See color version of this figure at back of this issue.

Figure 10. The VV-polarized measured and computed BRCS results for $\alpha=80^{\circ}$ incidence. See color version of this figure at back of this issue.

serves to validate and testifies to the accuracy of both measured and computed results. A scrutinized inspection of the Figures 7-14 reveals also the following points:

[20] 1. The relative RMS error between the measured and computed results is obtained for all frequencies and for all scattering angles using

$E_{R M S}=\sqrt{\frac{1}{N_{f} N_{\phi}} \sum_{i=1}^{N_{f}} \sum_{j=1}^{N_{\phi}}\left|\sigma_{M E A S}\left(f_{i}, \phi_{j}\right)-\sigma_{C O M P}\left(f_{i}, \phi_{j}\right)\right|^{2}}$,

where $N_{f}$ is the number of frequency samples and $N_{\phi}$ is the number of bistatic angles. The relative RMS error is
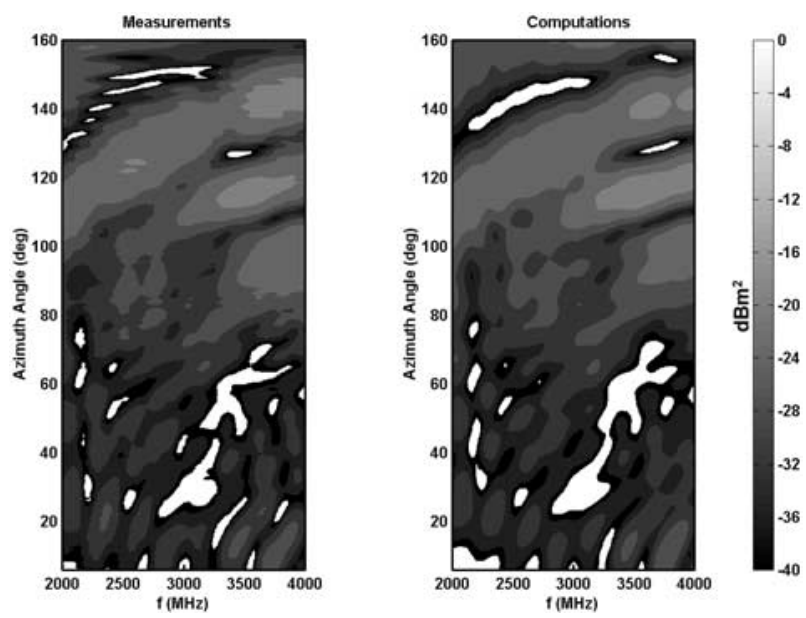

Figure 11. The HV-polarized measured and computed BRCS results for $\alpha=160^{\circ}$ incidence. See color version of this figure at back of this issue. 

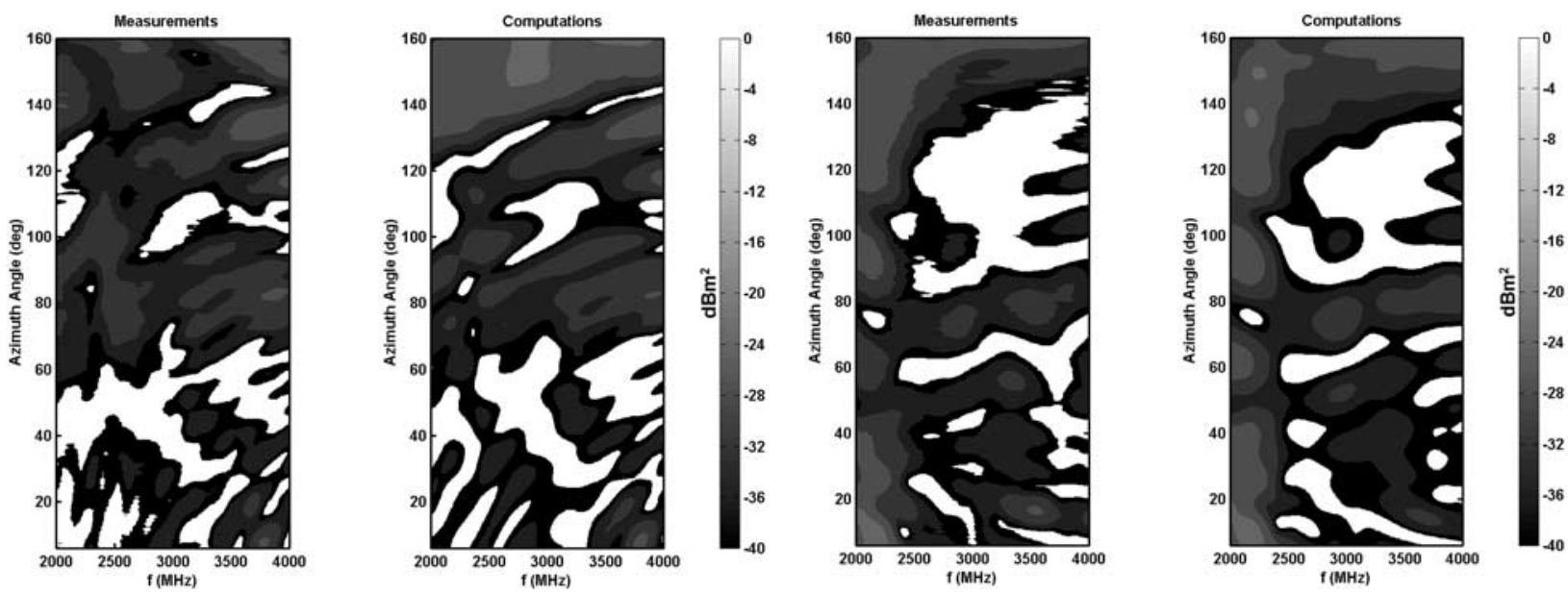

Figure 12. The VH-polarized measured and computed BRCS results for $\alpha=160^{\circ}$ incidence. See color version of this figure at back of this issue.

computed for each of one of the Figures 7-14, i.e., for each illumination angle and for each polarization. The results are tabulated in Table 1.

[21] 2. The white areas in the RCS contour plots, which correspond to values of RCS less than -40 $\mathrm{dBm}^{2}$, are in good agreement between the measured and computed results. This means that in addition to large values of RCS of the order of $1 \mathrm{~m}^{2}\left(0 \mathrm{dBm}^{2}\right)$, small values of the order of $10^{-4} \mathrm{~m}^{2}\left(-40 \mathrm{dBm}^{2}\right)$ are also in agreement. In other words, 4 orders of dynamic range of accuracy exist in both measured and computed results. This is especially important to validate the computational
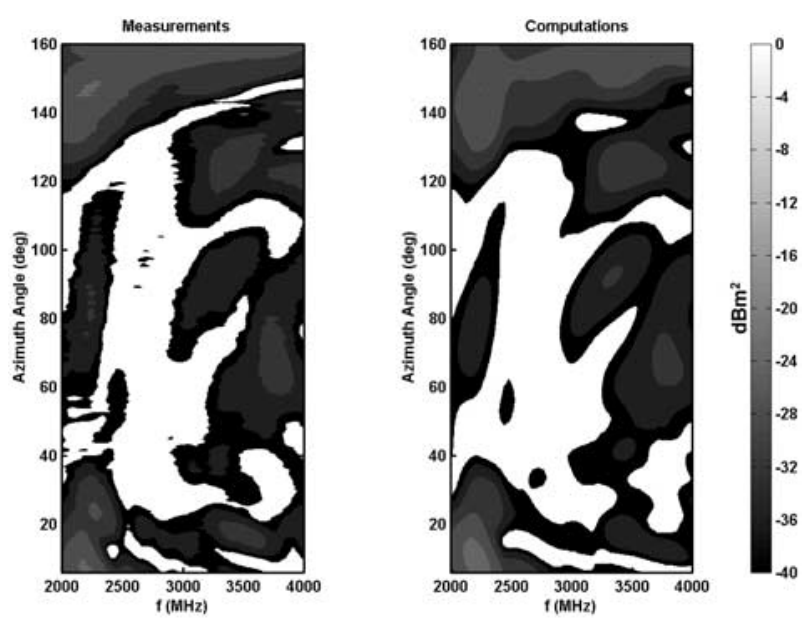

Figure 13. The HV-polarized measured and computed BRCS results for $\alpha=80^{\circ}$ incidence. See color version of this figure at back of this issue.

Figure 14. The VH-polarized measured and computed BRCS results for $\alpha=80^{\circ}$ incidence. See color version of this figure at back of this issue.

results since it shows that the accuracy is not compromised by using the FMM, which is an accelerated solution technique but does not necessarily jeopardize the accuracy.

[22] 3. Figure 15 presents the BRCS values of all polarizations as function of the scattering angle at 3 $\mathrm{GHz}$. The general agreement of the measured and computed results is quite good. The relative RMS error, as defined in equation (18), but for $N_{f}=1$, is 2.7, 1.8, 3.8, and $5.0 \mathrm{~dB}$ for $\mathrm{HH}, \mathrm{VV}, \mathrm{HV}$, and $\mathrm{VH}$ polarizations, respectively. Some of the minor disagreements visible in Figure 15 can be attributed to the manufacturing imperfections of the scaled model and also to the measurement artifacts as explained below.

[23] 4. In most of the measured results, small oscillations can be observed near the forward scattering directions where $\beta=140^{\circ}-160^{\circ}$, especially for the VV polarization. As seen in Figure 15, these small fluctuations are not present in the computed results. These artifacts are the result of the difficulty in decomposing the scattered and the incident signals, which are overlapping at the receiver near the forward scattering angles. In other words, near the forward scattering direction, the

Table 1. Relative RMS Error (dB) of the BRCS Values Presented in Figures 7-14

\begin{tabular}{ccccc}
\hline & \multicolumn{4}{c}{ Polarization } \\
\cline { 2 - 5 } Illumination & HH & VV & HV & VH \\
\hline$\alpha=160^{\circ}$ & 3.4 & 2.2 & 3.2 & 4.5 \\
$\alpha=80^{\circ}$ & 3.9 & 3.2 & 3.8 & 4.1 \\
\hline
\end{tabular}



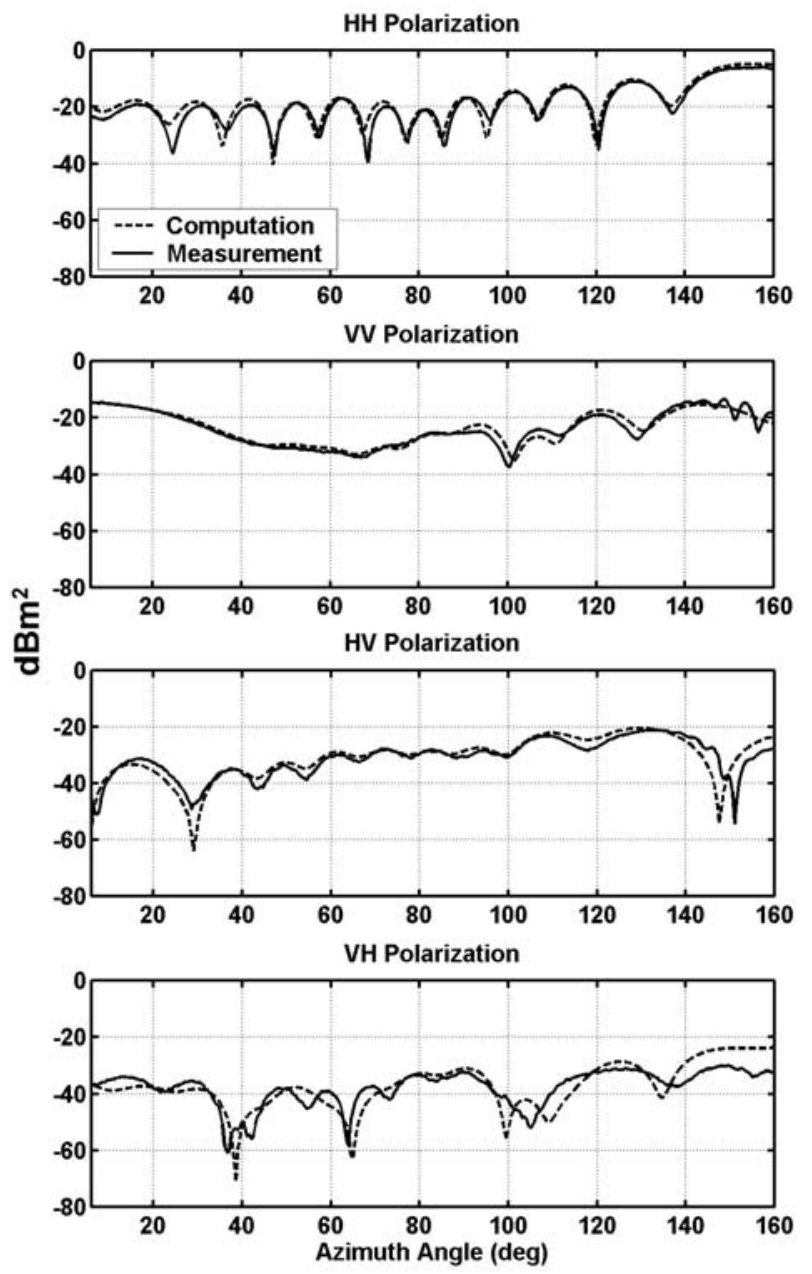

Figure 15. Measured and computed BRCS results for $\alpha=160^{\circ}$ incidence at $3 \mathrm{GHz}$.

receiving antenna receives a significant amount of incident field in addition to the scattered field. The performance of the coherent background subtraction that must cancel the incident field is limited by the dynamic range of the receiver. Therefore it is inevitable that a small amount of parasitic incident field remains and interferes with the scattered field to produce an amplitude modulation versus bistatic angle. This is clearly an artifact created by the measurement.

[24] The overall good agreement between the computations and measurements helps in building confidence in both methods toward further RCS predictions in the future.

\section{Conclusion}

[25] In this paper, BRCS values of a stealth airborne target are predicted. Predictions are performed by means of scaled-model measurements and numerical simulations. The FMM is implemented in the simulation environment so that large-scale CEM problems can be solved both efficiently and accurately. The remarkably good agreement between the measured and computed RCS results is interpreted as a validation of both of the prediction techniques.

[26] Acknowledgments. This work was sponsored by NATO AC323/SET036/TG21 (Impact of Emerging Technologies on Air Defence Radars) and is published with its authorization. This work was also supported by the Bilkent University Research Fund EE-01-01 and by the Turkish Academy of Sciences in the framework of the Young Scientist Award Program (LG/TÜBA-GEBİP/2002-1-12).

\section{References}

Bhattacharyya, A. K., and D. L. Sengupta, Radar Cross Section Analysis and Control, Artech House, Norwood, Mass., 1991.

Blyakhman, A. B., and I. A. Runova, Bistatic radar cross section and the detection of objects from their forward scatter, J. Commun. Technol. Electron., 46(4), 393-401, 2001.

Boyle, R. J., and W. Wasylkiwskyj, Comparison of monostatic and bistatic bearing estimation performance for low RCS targets, IEEE Trans. Aerosp. Electron. Syst., 30(3), 962968, 1994.

Castelli, J. C., BABI: An indoor bistatic RCS measurement facility-An overview of its performance, in Colloque International sur le Radar-International Conference on Radar, Paris, 3-6 May 1994, pp. 167-172, La Soc. de l'Electr. et de l'Electron., Paris, 1994.

Coifman, R., V. Rokhlin, and S. Wandzura, The fast multipole method for the wave equation: A pedestrian prescription, IEEE Antennas Propag. Mag., 35(3), 7-12, 1993.

Glasier, J. I., Some results in the bistatic radar cross section (RCS) of complex objects, Proc. IEEE, 77(5), 639-648, 1989.

Gürel, L., and H. Bağc1, Fast-multipole-method solution of the electromagnetic scattering from a stealth geometry, research report, Dep. of Electr. and Electron. Eng., Bilkent Univ., Ankara, Turkey, July 2001.

Gürel, L., K. Sertel, and İ. K. Şendur, On the choice of basis functions to model surface electric current densities in computational electromagnetics, Radio Sci., 34(6), 1373-1387, 1999.

Hanle, E., Survey of bistatic and multistatic radar, IEE Proc., Part F, 133(7), 587-595, 1986.

Howland, P. E., Target tracking using television-based bistatic radar, IEE Proc. Radar Sonar Navig., 146(3), 166-174, 1999.

Knott, E. F., Radar Cross Section Measurements, Van Nostrand Reinhold, New York, 1993.

Mie, G., Beiträge zur optik trüber medien, speziell kolloidaler metallösungen, Ann. Phys. Leipzig, 25, 377 pp., 1908.

Poullin, D., and M. Lesturgie, Multistatic radar using no cooperative transmitters, in Colloque International sur le Radar- 
International Conference on Radar, Paris, 3-6 May 1994, pp. 370-375, La Soc. de l'Electr. et de l'Electron., Paris, 1994.

Rao, S. M., D. R. Wilton, and A. W. Glisson, Electromagnetic scattering by surfaces of arbitrary shape, IEEE Trans. Antennas Propag., 30, 409-418, May 1982.

Rokhlin, V., Rapid solution of integral equations of scattering theory in two dimensions, J. Comput. Phys., 86, 414-439, 1990.

Sarabandi, K., F. T. Ulaby, and M. A. Tassoudji, Calibration of polarimetric radar systems with good polarisation isolation, IEEE Trans. Geosci. Remote Sens., 28(1), 70-75, 1990.

Sheng, X. Q., J.-M. Jin, J. Song, W. C. Chew, and C.-C. Lu, Solution of combined-field integral equation using multilevel fast multipole algorithm for scattering by homogeneous bodies, IEEE Trans. Antennas Propag., 46(11), 1718-1726, 1998.

Skolnik, M., (Ed.), Radar Handbook, McGraw-Hill, New York, 1991.

Song, J., C.-C. Lu, and W. C. Chew, Multilevel fast multipole algorithm for electromagnetic scattering by large complex objects, IEEE Trans. Antennas Propag., 45(10), $1488-$ 1493, 1997.
Stone, W. R., (Ed.), Radar Cross Sections of Complex Objects, IEEE Press, Piscataway, N. J., 1989.

Titin-Schnaider, C., P. Dreuillet, and J. F. Pétex, A polarimetric tool for radar target analysis, in Colloque International sur le Radar-International Conference on Radar, Paris, 3-6 May 1994, pp. 596-601, La Soc. de l'Electr. et de l'Electron., Paris, 1994.

Ufimtsev, P. Y., Comment on diffraction principles and limitations of RCS reduction techniques, Proc. IEEE, 84(12), 1830-1851, 1996.

Winchester, T. A., Pulsed radar return from a chaff cloud, IEE Proc., Part F, 139(4), 315-320, 1992.

H. Bağc1 and L. Gürel, Department of Electrical and Electronics Engineering, Bilkent University, TR-06800 Bilkent, Ankara, Turkey. (lgurel@ee.bilkent.edu.tr)

J. C. Castelli, A. Cheraly, and F. Tardivel, Electromagnetism and Radar Department, Office National d'Etudes et de Recherche Aérospatiale (ONERA), BP 72 92322, Châtillon Cedex, France. 

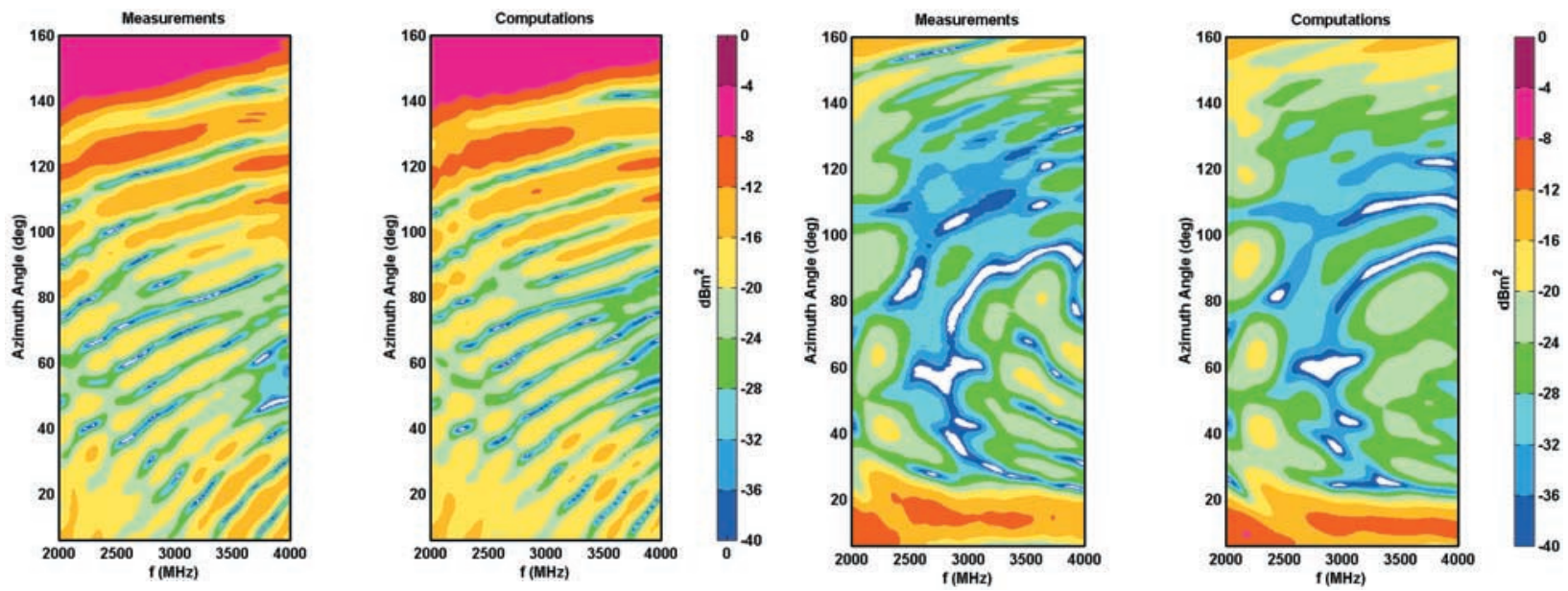

Figure 7. The HH-polarized measured and computed BRCS results for $\alpha=60^{\circ}$ incidence.

Figure 9. The HH-polarized measured and computed BRCS results for $\alpha=80^{\circ}$ incidence.
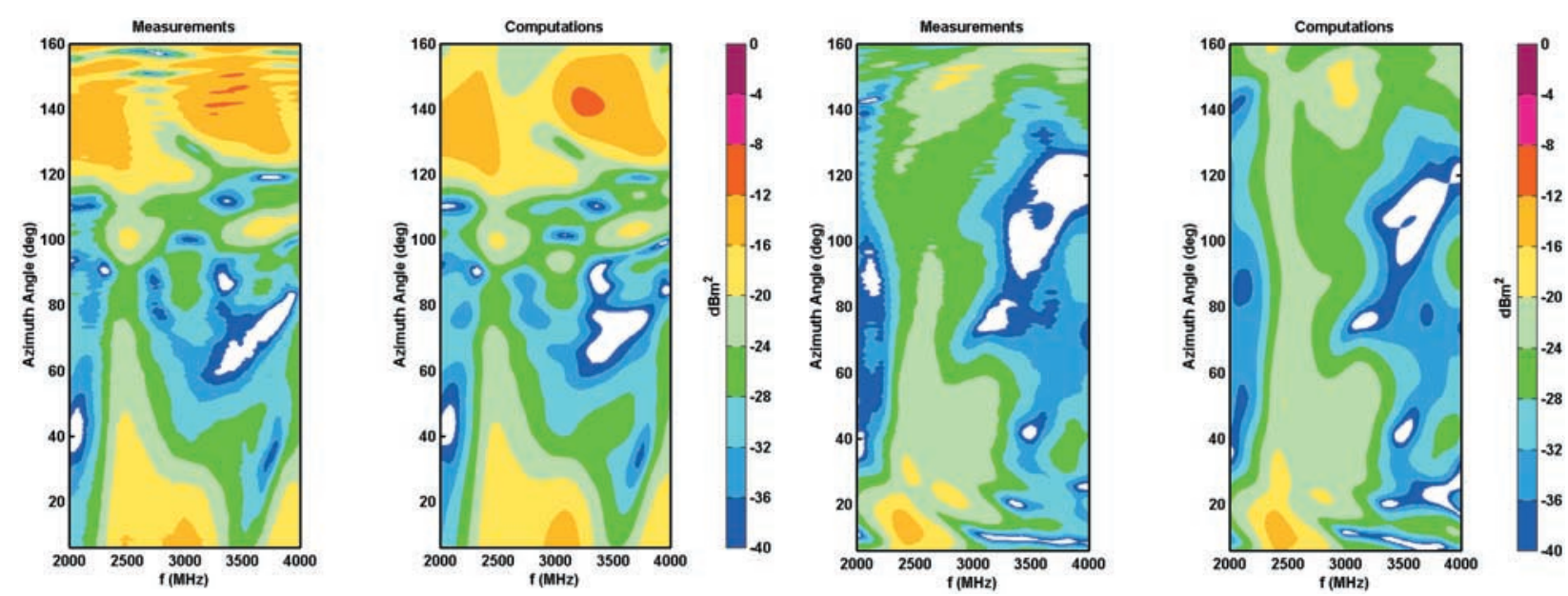

Figure 8. The VV-polarized measured and computed BRCS results for $\alpha=160^{\circ}$ incidence.

Figure 10. The VV-polarized measured and computed BRCS results for $\alpha=80^{\circ}$ incidence. 

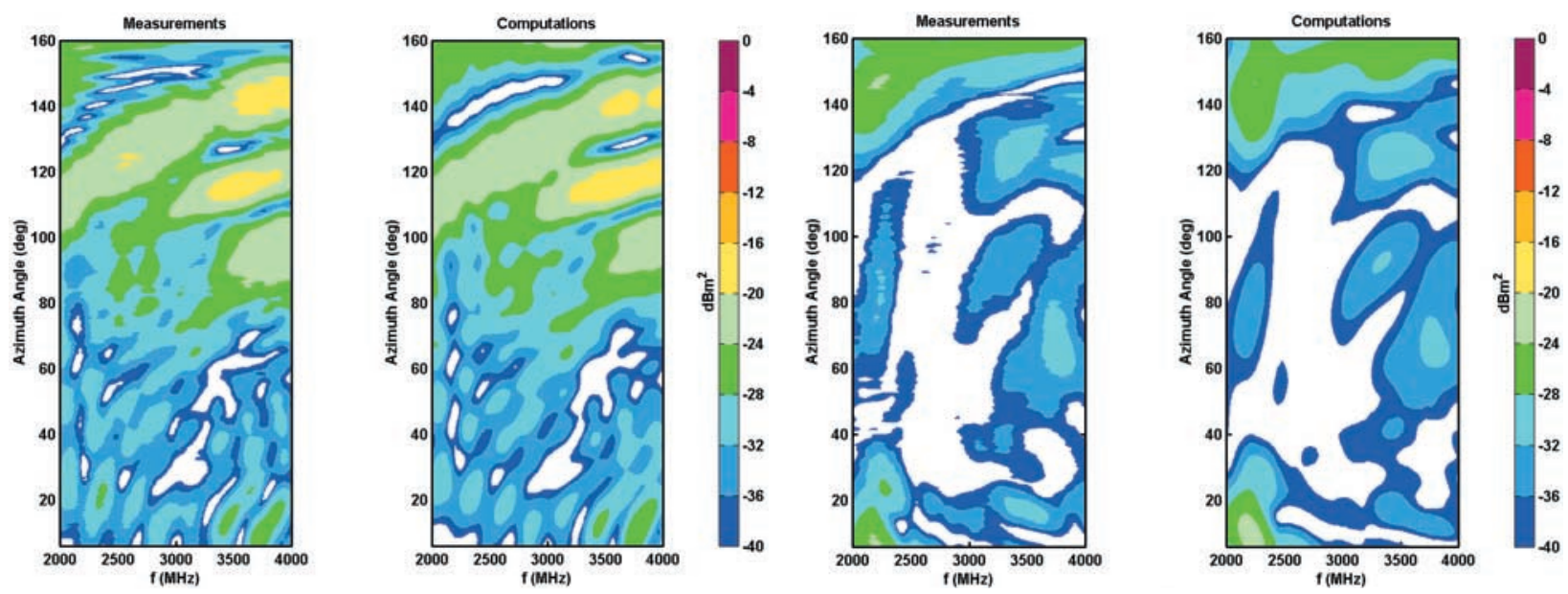

Figure 11. The HV-polarized measured and computed BRCS results for $\alpha=160^{\circ}$ incidence.

Figure 13. The HV-polarized measured and computed BRCS results for $\alpha=80^{\circ}$ incidence.
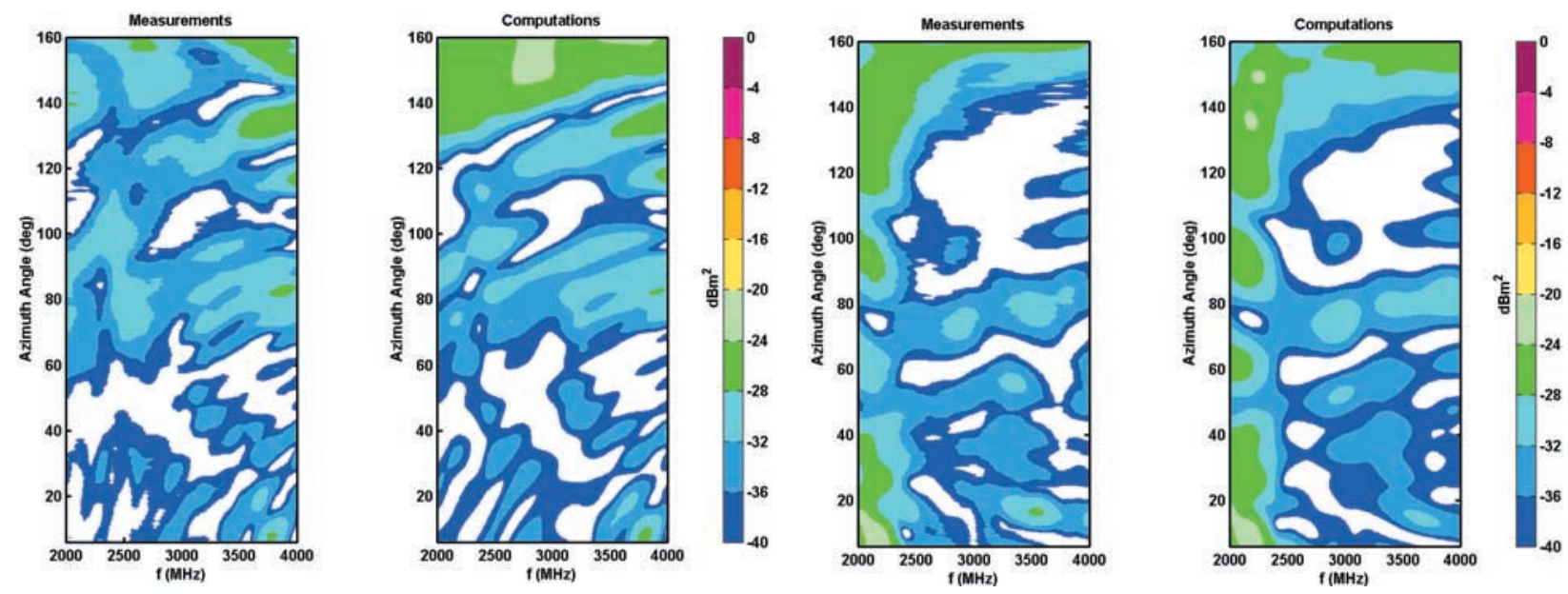

Figure 12. The VH-polarized measured and computed BRCS results for $\alpha=160^{\circ}$ incidence.

Figure 14. The VH-polarized measured and computed BRCS results for $\alpha=80^{\circ}$ incidence. 